\title{
Temperature-dependent structure of molten eutectic $\mathrm{Cu}_{37} \mathrm{Sb}_{63}$
}

\author{
Guohua Ding ${ }^{1 *}$, Limeng Liang ${ }^{1}$ \\ ${ }^{1}$ School of Mechanical and Vehicular Engineering, Bengbu University, Bengbu 233030, China;
}

\begin{abstract}
Quoted measurements of viscosity and our results of electrical resistivity experiments indicate two anomalous changes of physical properties take place in molten eutectic $\mathrm{Cu}_{37} \mathrm{Sb}_{63}$. Effective pair inter-ionic potentials evaluated using the hyper-netted chain theory further confirms the presence of two discontinuous structural transitions within the ranges from $1273 \mathrm{~K}$ to $1073 \mathrm{~K}$ and from $1073 \mathrm{~K}$ to $903 \mathrm{~K}$, respectively. Moreover, the result of electrical resistivity experiments suggests the two structural transitions for molten $\mathrm{Cu}_{37} \mathrm{Sb}_{63}$ are reversible.
\end{abstract}

\section{Introduction}

The nature of viscous liquids is recognized as an important but unsolved problem in condensed state physics [1,2]. For decades, many experimental and theoretical studies [3-7] have proven that liquid structure and various physical properties change discontinuously at hundreds of degrees above liquidus, as a function of temperature at constant pressure in liquid metals and alloys. Moreover, the characteristic of liquid metals or melts will have a strong impact on the qualities of the solid materials [8].

$\mathrm{Cu}-\mathrm{Sb}$ alloys are applied extensively in material, electricity, and especially in the field of functional composite materials because of their excellent electrical and chemical properties. Much attention has been paid to the physical properties and structure of $\mathrm{Cu}-\mathrm{Sb}$ alloys. In a previous work $\mathrm{Cu}-\mathrm{Sb}$ molten alloys have been investigated [9]. For the eutectic $\mathrm{Cu}_{37} \mathrm{Sb}_{63}$ alloy, segregation into clusters corresponding to an association of Sb-atoms and association of unlike atoms was found.

In order to gain an insight into the temperature-dependent structure and physical properties of molten eutectic $\mathrm{Cu}_{37} \mathrm{Sb}_{63}$, we measured the electrical resistivity and calculated effective pair inter-ionic potentials evaluated using the hyper-netted chain theory to obtain understanding of its structural changes. Noted that, the morphological changes of calculated effective pair potentials are consistent with the turning point of the experimental electrical resistivity and the quoted viscosity.

\section{Experimental}

The measurements of viscosity $(\eta)$ for molten eutectic $\mathrm{Cu}_{37} \mathrm{Sb}_{63}$ were carried out using an oscillating cup viscometer by Ning et al. [10]. The samples, after maintaining for 3 hours at $1240 \mathrm{~K}$, were cooled down to the experimental temperatures with a rate of $4 \mathrm{~K} / \mathrm{min}$ and held for 1 hour before each measurement. Figure 1 shows the Arrhenius plots $(\ln \eta \sim 1000 / T)$ for molten eutectic $\mathrm{Cu}_{37} \mathrm{Sb}_{63}$. Generally, the viscosity behavior of the melt obeys the Arrhenius equation [11] within a temperature zone:

$$
\eta=\eta_{0} \exp \left(\frac{E_{v}}{R T}\right)
$$

where $\eta_{0}$ is a pre-exponential constant, which is associated with the nature of the liquid, $R$ is the molar gas constant and $E_{v}$ is the viscous-flow activation energy. Viscosity is known to be one of the most structure-sensitive properties. As shown in figure 1, it is obvious that there is an abrupt change for measured viscosity at $1160 \mathrm{~K}$ and $944 \mathrm{~K}$, respectively, which imply twice structural transitions may occur at the location of discontinuous point. 


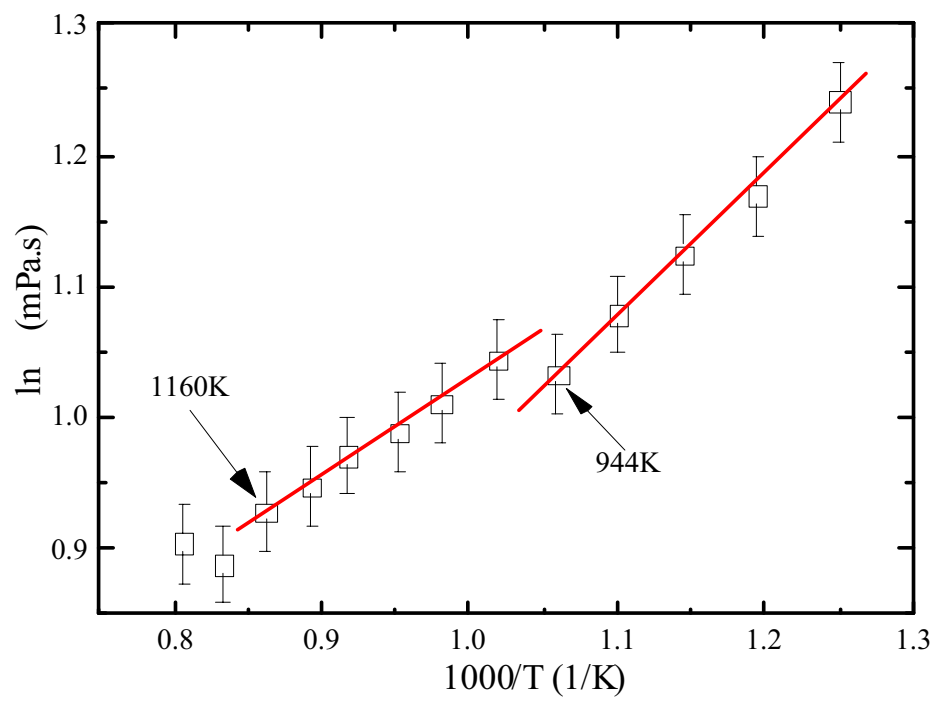

Figure 1. Temperature dependence of viscosity for molten $\mathrm{Cu}_{37} \mathrm{Sb}_{63}$ at the cooling process. The lines are guides to the eyes.

Electrical resistivity $(\rho)$ is also sensitive to liquid structure. Measurement of electrical resistivity for molten eutectic $\mathrm{Cu}_{37} \mathrm{Sb}_{63}$ was carried out by D.C. four-probe method. Four tungsten electrodes $1 \mathrm{~mm}$ in diameter were placed in the wall of the cell along its vertical axis for voltage measurements under a constant current. The voltage was measured by a KEITHLEY-2182 nanovoltmeter while a constant current of $500 \mathrm{~mA}$ was supplied by a PF66M sourcemeter. Two Ni-Cr and Ni-Si thermocouples were used for temperature measurements. The experimental details, as well as the experimental equipment, were described in Ref. [12]. The heating and cooling rates were both set to $5 \mathrm{~K} / \mathrm{min}$ in the experimental cycles. During the experiments, the samples were protected from oxidation by a purified argon medium. By repeating the measurements, a reproducible set of data was obtained and the experiment errors were found to be within $\pm 3 \%$.

\section{Results and discussion}

The resistivity versus temperature plot of molten $\mathrm{Cu}_{37} \mathrm{Sb}_{63}$ at the first cooling process is shown in figure 2. As the temperature decreased, the resistivity linearly decreases within the range from $1373 \mathrm{~K}$ to $1103 \mathrm{~K}$ and from $953 \mathrm{~K}$ to the starting point of solidification. Noted that, the resistivity-temperature curve shows twice anomalous changes at $1103 \mathrm{~K}$ and $953 \mathrm{~K}$, respectively, which are in good agreement with the discontinuous points of viscosity.

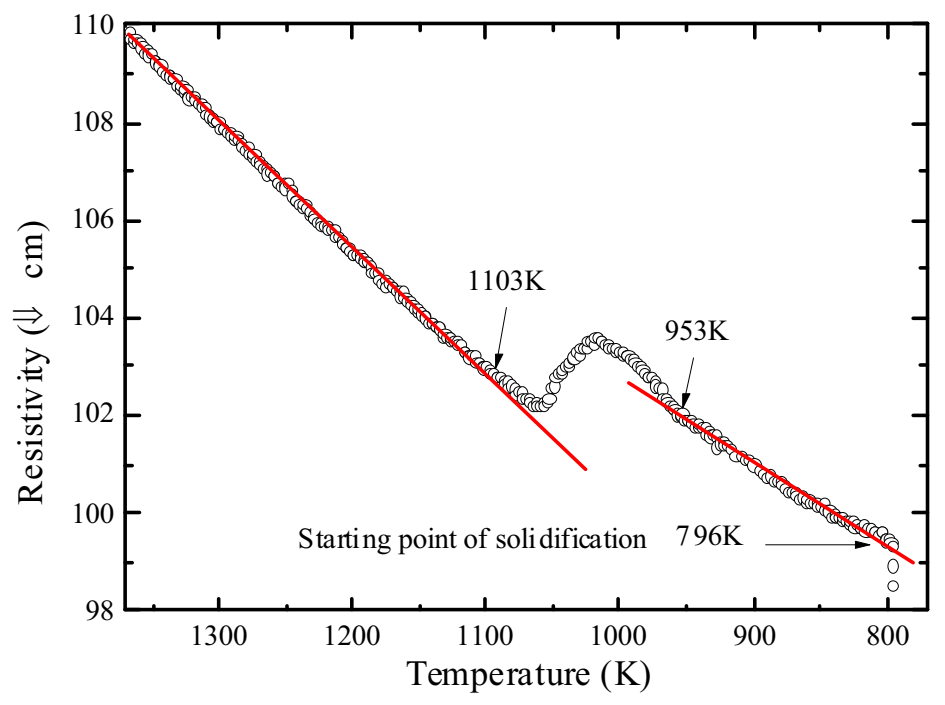

Figure 2. Resistivity-temperature curve of molten $\mathrm{Cu}_{37} \mathrm{Sb}_{63}$ at the first cooling process. The lines are guides to the eyes.

In the study of various properties of liquid metals or alloys, the knowledge of the interaction between the component ions is frequently required. At present time, the effective pair inter-ionic potentials of molten $\mathrm{Cu}_{37} \mathrm{Sb}_{63}$ at different temperatures were evaluated using the hyper-netted chain theory (HNC) from the static structure factor observed by X-ray diffraction experiments [13]. In the HNC approximation, the effective pair potential $V(r)$ is given by 


$$
V(r)=k_{B} T[g(r)-c(r)-1-\ln g(r)]
$$

where $k_{B}$ is the Boltzmann factor, $T$ is the temperature in degrees Kelvin, $g(r)$ is the pair distribution function and $c(r)$ is the direct correlation function. The direct correlation function is calculated by using equation (3).

$$
c(r)=\frac{1}{2 \pi^{2} \rho_{0} r} \int_{0}^{\infty}\left[\frac{S(Q)-1}{S(Q)}\right] Q \sin (Q r) d Q
$$

Noted that, the low $Q$ portion of $S(Q)$ is necessary with high accuracy because the static structure factor appears in the denominator of the integrand for $c(r)$. In this paper, the magnitude of the diffraction vector $Q$ ranged from 0.05 to $12.25 \AA-1$.

Figure 3 shows the effective pair inter-ionic potentials of molten $\mathrm{Cu}_{37} \mathrm{Sb}_{63}$ at different temperatures. It is obvious that the pair potential $V(r)$ in molten $\mathrm{Cu}_{37} \mathrm{Sb}_{63}$ has a temperature sensitive long-range oscillatory type potential. The oscillations of the tail of the inter-ionic potential are due to the free motion of the valence electrons between cores. As shown in figure 3, the pair potentials for molten $\mathrm{Cu}_{37} \mathrm{Sb}_{63}$ at $1073 \mathrm{~K}$ and $1273 \mathrm{~K}$ are characterized by a fairly large repulsive positive barrier, while at $903 \mathrm{~K}$ the long range oscillations of the inter-ionic potential almost wane, which suggests that the free motion of the valence electrons becomes limited with temperature decreasing and they have been localizing around their parent ions.

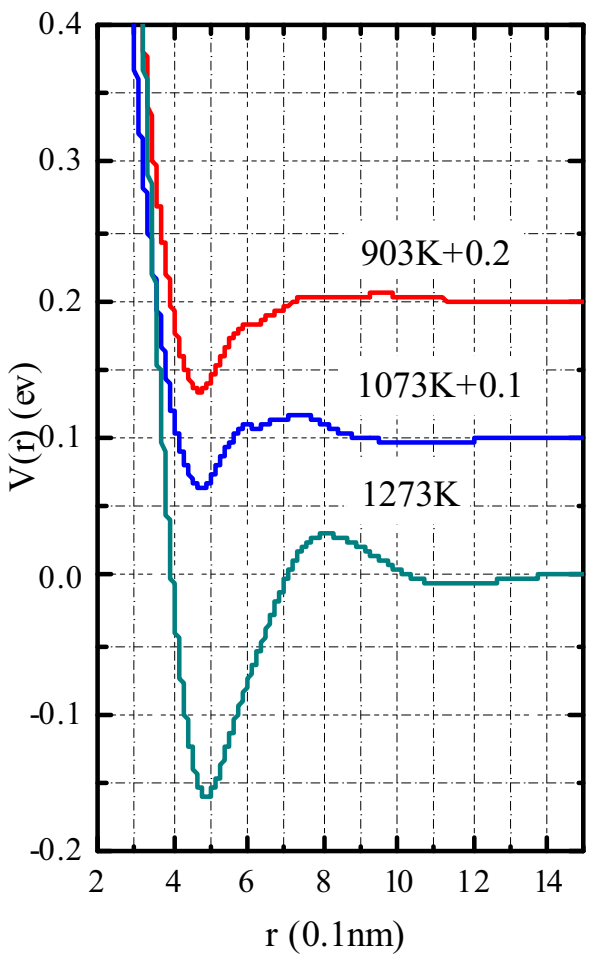

Figure 3. Effective pair inter-ionic potentials for molten $\mathrm{Cu}_{37} \mathrm{Sb}_{63}$ at different temperatures.

Moreover, the shapes of the oscillatory characteristics at $903 \mathrm{~K}$ and $1073 \mathrm{~K}$ are same to those of semi-metallic liquids, such as liquid $\mathrm{Sb}[14], \mathrm{Bi}[14,15]$ and $\mathrm{Ga}[16]$. But the pair potential at $1273 \mathrm{~K}$ seems to show remarkably the long-range oscillatory characteristics expected for metallic liquids [17]. These results suggest that molten $\mathrm{Cu}_{37} \mathrm{Sb}_{63}$ has a high concentration of "free electrons" at higher temperature, as pointed out by Waseda et al. about liquid Te [14], which correspond to the pronounced increase of the resistivity of molten $\mathrm{Cu}_{37} \mathrm{Sb}_{63}$ at $1073 \mathrm{~K}$ at the cooling process as shown in figure 2. The pair potential for liquid $\mathrm{Cu}_{37} \mathrm{Sb}_{63}$ at $1273 \mathrm{~K}$, shown in figure 3 , is characterized by a fairly large attractive negative barrier. This diversion of the potential well-depth of the potential function can be related to the change in the nature of the interactions between particles.

Therefore, the applied potential model in this work can predict the two structural transitions for molten $\mathrm{Cu}_{37} \mathrm{Sb}_{63}$ within the ranges from $1273 \mathrm{~K}$ to $1073 \mathrm{~K}$ and from $1073 \mathrm{~K}$ to $903 \mathrm{~K}$, respectively, which are in good agreement with the discontinuous points of viscosity and electrical resistivity.

It should be pointed out that the two structural transitions for molten $\mathrm{Cu}_{37} \mathrm{Sb}_{63}$ are reversible. As shown in figure 4 , all the $\rho$-T curves present reversible changing characters in subsequent two heating and cooling cycles. Although the turning points at the cooling process are different from those at the heating process, which might result from the hysteresis effect on electrical resistivity of melt superheat, this figure presents very good reversibility in heating and cooling cycles.

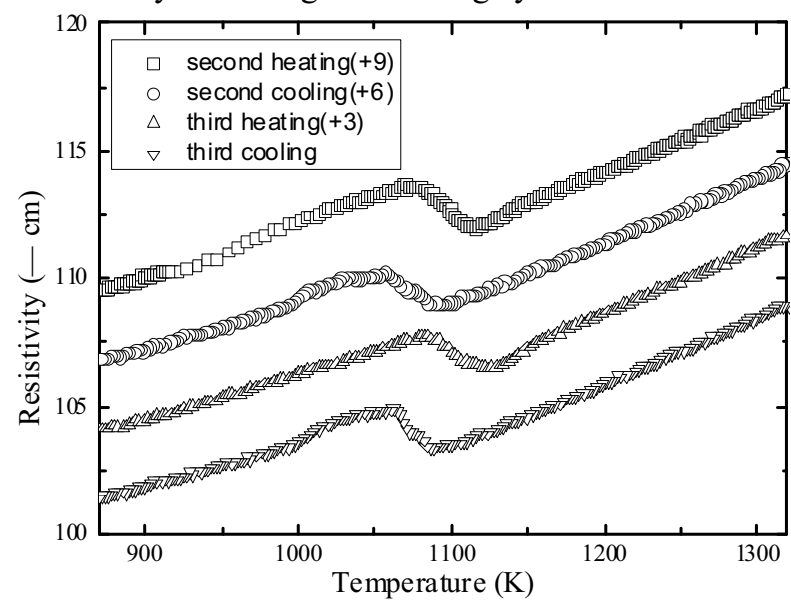

Figure 4. Resistivity-temperature curves of molten $\mathrm{Cu}_{37} \mathrm{Sb}_{63}$ in subsequent two heating and cooling cycles.

To explain the two structural transitions we propose the following scheme. The low temperature liquid is inhomogeneous, and it is decomposed into regions which are built up from an association of Sb-atoms and association of unlike atoms [18]. Essentially speaking, there are still two different kinds of phases (L'+L') in the melts. They are, however, metastable and prone to homogenization. As temperature is elevated, the atoms within the associations gain higher and higher energy. Once the critical temperature is reached, the energy is high enough to overcome the energy barrier so that the $\mathrm{Sb}-\mathrm{Sb}$ covalent bond in original phases is broken continuously. At the same time, the new $\mathrm{Cu}-\mathrm{Sb}$ atomic bonds build up, by which the relatively homogeneous liquid phase (L) nucleates and grows. That is, the phase transition $\mathrm{L}^{\prime}(\mathrm{mSb})+\mathrm{L}{ }^{\prime}\left(\mathrm{nCu}_{2} \mathrm{Sb}\right) \rightarrow \mathrm{L}\left(\mathrm{Cu}_{2 \mathrm{n}} \mathrm{Sb}_{\mathrm{m}+\mathrm{n}}\right)$ takes place. 


\section{Conclusions}

Analyses of viscosity, electrical resistivity and effective pair inter-ionic potentials obtained from molten $\mathrm{Cu}_{37} \mathrm{Sb}_{63}$ showed that with decreasing temperature two discontinuous structural transitions within the ranges from $1273 \mathrm{~K}$ to $1073 \mathrm{~K}$ and from $1073 \mathrm{~K}$ to $903 \mathrm{~K}$, respectively, takes place. The morphological changes of the pair potential curves indicate that the valence electrons have been localizing around their parent ions at $903 \mathrm{~K}$, while molten $\mathrm{Cu}_{37} \mathrm{Sb}_{63}$ has a high concentration of "free electrons" at $1273 \mathrm{~K}$. Such representation of the structure of molten $\mathrm{Cu}_{37} \mathrm{Sb}_{63}$ explains the peculiar temperature dependences of electrical resistivity. Moreover, the result of electrical resistivity experiments suggests the two structural transitions for molten $\mathrm{Cu}_{37} \mathrm{Sb}_{63}$ are reversible.

\section{Acknowledgements}

This work was financially supported by the Key Project in the Youth Elite Support Plan in Universities of Anhui Province (Grant No. gxyqZD2018084) and Scientific Research Foundation for Talented Scholars of Bengbu University (Grant No. BBXY2018KYQD01). We thank Prof. Walter Hoyer for sharing S(q) data of molten eutectic $\mathrm{Cu}_{37} \mathrm{Sb}_{63}$.

\section{References}

1. Anderson, P.W.: Science, 267, 1995, p. 1615-1616.

2. Sokolov, A.P.: Science, 273, 1996, p. 1675-1676.

3. Franzese, G., Malescio, G., Skibinsky, A., Buldyrev, S.V., Stanley, H.E.: Nature, 409, 2001, p. 692.

4. Zu, F.Q., Zhu, Z.G., Guo, L.J., Qin, X.B., Yang, H., Shan, W.J.: Phys. Rev. Lett., 89, 2002, p. 125505.

5. Kurita, R., Murata, K.-I., Tanaka, H.: Nat. Mater., 7, 2008, p. 647-652.

6. Greenberg, Y., Yahel, E., Caspi, E.N., Benmore, C., Beuneu, B., Dariel, M.P., Makov, G.: Europhys. Lett., 86, 2009, p. 36004.

7. Bian, X.F., Wang, W.M.: Mater. Lett., 44, 2000, p. 54-58.

8. Bian, X.F., Guo, J., Lv, X.Q., Qin, X.B., Wang, C.D.: Appl. Phys. Lett., 91, 2007, p. 221910.

9. Halm, T., Neumann, H., Hoyer, W.: Z. Naturforsch. A, 49, 1994, p. 530-534.

10. Ning, S., Bian, X.F., Ren, Z.F.: Physica B, 405, 2010, p. 3633-3637.

11. Chhabra, R.P., Sheth, D.K.: Z. Metallkd., 81, 1990, p. 264-271.

12. Li, X.F., Zu, F.Q., Ding, H.F., Yu, J., Liu, L.J., Li, Q., Xi, Y.: Physica B, 358, 2005, p. 126.

13. http://www.tu-chemnitz.de/physik/RND/structquery. php.en.

14. Waseda, Y., Suzuki, K.: Phys. Stat. Sol. B, 51, 1972, p. K109-K112.
15. Dahlborg, U.: Phys. Chem. Liq., 15, 1986, p. 243-252.

16. Schommers, W.: Phys. Rev. A, 28, 1983, p. 3599.

17. Waseda, Y., Suzuki, K.: Phys. Stat. Sol. B, 49, 1972, p. 643-650.

18. Kaban, I., Halm, T., Hoyer, W.: J. Non-Cryst. Solids, 288, 2001, p. 96-102. 